\title{
Landscape as a factor of natural selection on reactivity for steppe marmot (Marmota bobak)
}

\author{
Alexander Nikol'skii* \\ Peoples Friendship University of Russia (RUDN University), Faculty of Ecology, 6 Miklukho- \\ Maklaya Street, Moscow, 117198, Russian Federation
}

\begin{abstract}
Marmots use a sound signal to inform their neighbours about the danger. Vocalization is the response of marmots to the visual perception of a potentially dangerous stimulus. For the first time at the intraspecific level the relationship between the rhythmic structure of the signal and the habitat elevation of populations is shown using the example of the steppe marmot (Marmota bobak). An increase in elevation is accompanied by an increase in the relative excess of positive forms over the nearest negative forms of relief. The dismembered relief reduces the visual detection range of danger. With an increase in the habitat elevation of populations, the repetition periods of sounds in the signal decrease. In the range of habitat elevations of the steppe marmot from 120 to $730 \mathrm{~m}$, the median of the repetition periods decreases from 5.35 to $2.17 \mathrm{~s}$. The increased rhythm is a consequence of the increased reactivity of marmots. It has been suggested that a complex of reactions to visual detection of danger controls the amygdale. The alarm call of marmots was recorded in the field.
\end{abstract}

\section{Introduction}

Changes in ecological factors can cause variability of communications means [1]. My report shows how a change in the landscape structure becomes a natural selection factor, influencing the variability of the rhythmic organization of the marmots' alarm call.

Marmots (Marmota, Rodentia), as well as other inhabitants of open landscapes with daytime activity, inform their neighbors about the danger by means of a sound signal. Vocalization is a behavioral response (reaction) of marmots to the visual perception of a potentially dangerous object. I use the concept of "reactivity" in the meaning accepted in the behavioral sciences, as a response to a stimulus [2].

An alarm call (here in after referred to as a signal) consists of a sequence of short sounds. The variability of the signal, intraspecific divergence and interspecific parallelisms, indirectly confirm the influence of natural selection on the process of forming its rhythmic organization. The rhythmic organization of the signal is ecologically determined by the dismemberment of the terrain, the relative excess of the positive forms of the earth's surface over the nearest negative forms. In habitats with dismembered terrain that restricts the view, marmots vocalize at an accelerated rate $[3,4]$.

*Correspondingauthor: bobak@list.ru 
At the interspecific level, the relationship between the rhythmic structure of the signal and the landscape can be detected without resorting to special analysis methods. Thus, the signal of the low elevation steppe marmot consists of a sequence of slowly following sounds, and in the signal of the typical mountain species, the red ( $M$. caudata) and Himalayan (M. himalayana) marmots [5], the sounds are grouped in rapid series (Fig. 1).
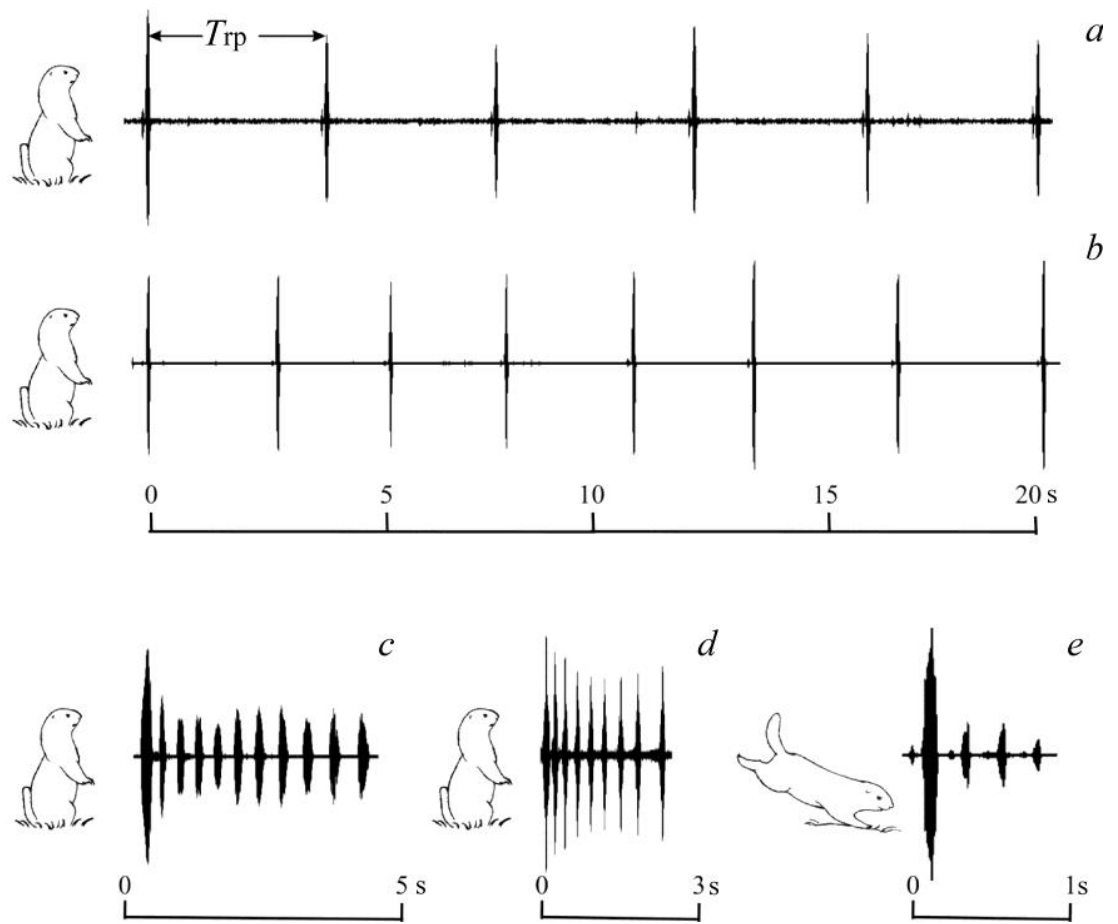

Fig. 1. Rhythmic structure of the alarm call of the steppe marmot: $a$ - local population "3", Ukraine, Kharkiv region, Russian Plain, the habitat elevation of the population is $170 \mathrm{~m} ; b$ - local population "8", Kazakhstan, Karaganda region, Kazakh Melkosopochnik, the habitat elevation of the population is $730 \mathrm{~m}$. Rhythmic structure of the alarm call of typical mountain species, the habitat elevation of the population is about $3000 \mathrm{~m}$ : $c$-signal of the red marmot (M. caudata); $d$-signal of the Himalayan marmot (M. himalayana). $e$-signal accompanying the escape of the steppe marmot into the burrow. $T_{\mathrm{rp}}$ - repetition period of sounds in the signal. In front of the oscillograms are shown the characteristic postures of marmots during the alarm call $(a-d)$ and a signal accompanying escape into a burrow $(e)$, poses of marmots.

Marmots react to the appearance of predators hundreds of meters away. As a result, the middle forms of relief, such as hills, hummocks, and cutters in the main slope, are commensurate with the range of danger detection by marmots. The dissected terrain reduces the range of visual detection of danger and increases the likelihood of encountering a potentially dangerous stimulus at an unexpectedly close distance (Fig. 2).

In this work, the relationship between the vocal activity of marmots and the landscape is shown for the first time at the intraspecific level. As an object of research, the steppe marmot (Marmota bobak Müller, 1776) was chosen as the only inhabitant of the hilly-rocky plains among the marmots of the Old World. All other species of the genus Marmota inhabit mountains [5], usually with strong and very strong vertical dismemberment. Also, for the first time, a visual model of the influence of the landscape on the variability of the reactivity of marmots, realized by them through vocalization, is constructed. 

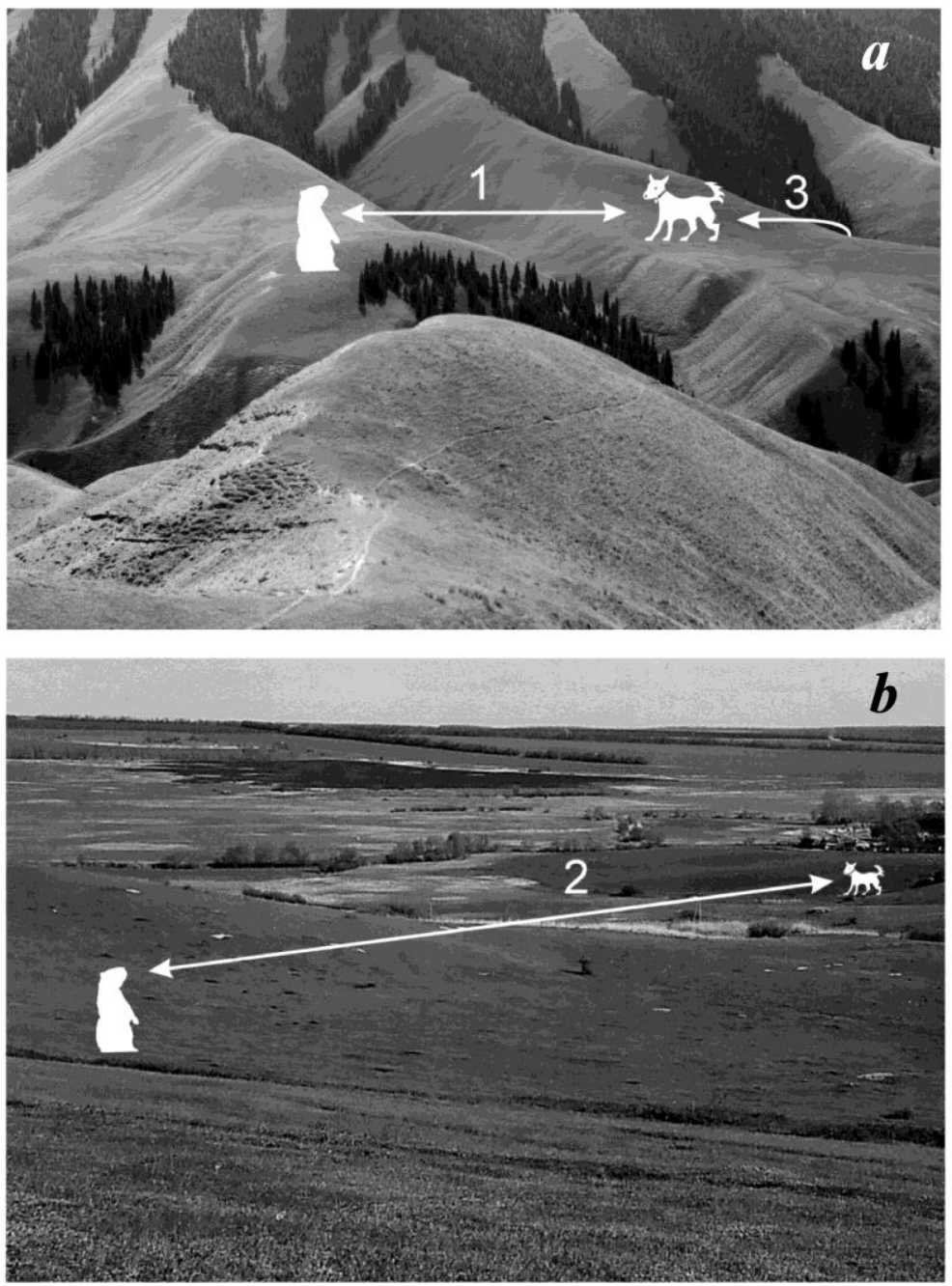

Fig. 2. Influence of landscape on visual detection by marmots of a potentially dangerous stimulus in the mountains ( $a$, Eastern Tien Shan, China, the range of the gray marmot, M. baibacina) and on the hilly-ridged plain ( $b$, Kharkiv region, Ukraine, the range of the steppe marmot, M. bobak). 1, 2 - the relative distance of a high probability of detection by marmots of a potentially dangerous stimulus; 3 - the trajectory of the unexpected appearance of a potentially dangerous stimulus in the mountains.

\section{Material and Methods}

The material was collected in the field. It includes 10 local populations of the steppe marmot, from the Kharkiv region in Ukraine to the Karaganda region in Kazakhstan (Fig. 3 ). The sound reaction of marmots was provoked by a person. During the recording on the tape recorder, the operator was at least a few tens of meters away from the marmot vocalizing in a vertical position (Fig. 1), which reduced the impact of situational changes in the signal on the results of observations. Each population is represented by 7 individuals older than 1 year. As a measure of the reactivity of marmots, the repetition period of sounds was taken, the time between the tops of adjacent sounds. The measurements were carried out using oscillograms $\left(T_{\text {rp }}\right.$, Fig. $\left.1: a\right)$. The shorter the repetition periods, the more often the sounds are repeated and, therefore, the higher the reactivity of the signal source realized through vocalization. In the signal of each individual, 5 periods were measured. To measure 
them, the SpectraPLUS program is used. Statistical processing of the results of observations was carried out in the program Statgraphics. $\mathrm{P} \leq 0.05$.

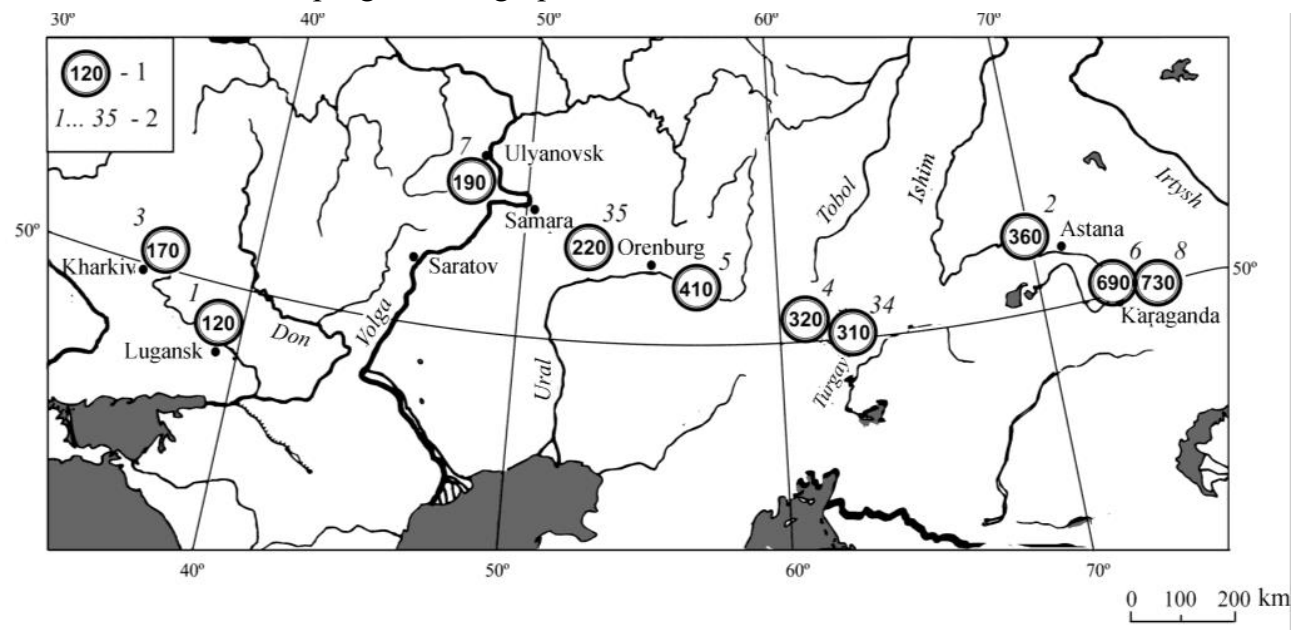

Fig. 3. Distribution of local populations of steppe marmot. 1 - habitat elevation of the population, $m$; 2 - registration numbers of local populations of the steppe marmot.

To characterize the landscape, I use the elevation above sea level, reading it in the Google Earth Pro program according to the geographical coordinates of the populations' habitats.

The absolute elevation affects the relative excess of the tops of positive forms over the nearest negative forms of relief [6]. The process of dismemberment of the relief is aggravated by secondary water and gravitational erosion [7]. In general, the higher the terrain, the deeper the relief is dissected.

\section{Results}

The results of the statistical analysis are shown in Fig. 4. I use two statistical methods: 1) the position of the median $(M e)$ of the repetition period of sounds $\left(T_{r p}\right)$ with a $95 \%$ confidence interval (Fig. $4: a$ ), and 2) regression analysis of the relationship between the repetition periods of sounds and the habitat elevation $(H)$ of the population (Fig. $4: b$ ).

In most populations, the distribution of repetition periods differs from the normal one, which affects the relative position of the median (Me, Fig. $4: a$ ). In the future, distribution analysis may be useful in describing the form of natural selection [8].

In the range of elevation of the steppe marmot' habitats from 120 to 730 m the periods of repetition are more than halved, from 5.35 to 2.17 seconds.

Regression analysis (Fig. $4: b$ ) confirmed the dependence of the repetition period of sounds on the habitat elevation of the population. The regression equation is:

$$
T_{\mathrm{rp}}=(1.45561+109.952 / H)^{2},
$$

where $T_{\mathrm{rp}}$ is the repetition period of sounds in the signal, $\mathrm{s} ; H$ is the habitat elevation of the population, $\mathrm{m}$. The coefficient of determination $\left(\mathrm{R}^{2}\right)$, equal to $65 \%$, indicates a moderately strong relationship between the repetition period of sound and elevation. The rate of reduction of the repetition periods is maximal within the first $250 \mathrm{~m}$ (Fig. $4: b$ ). 


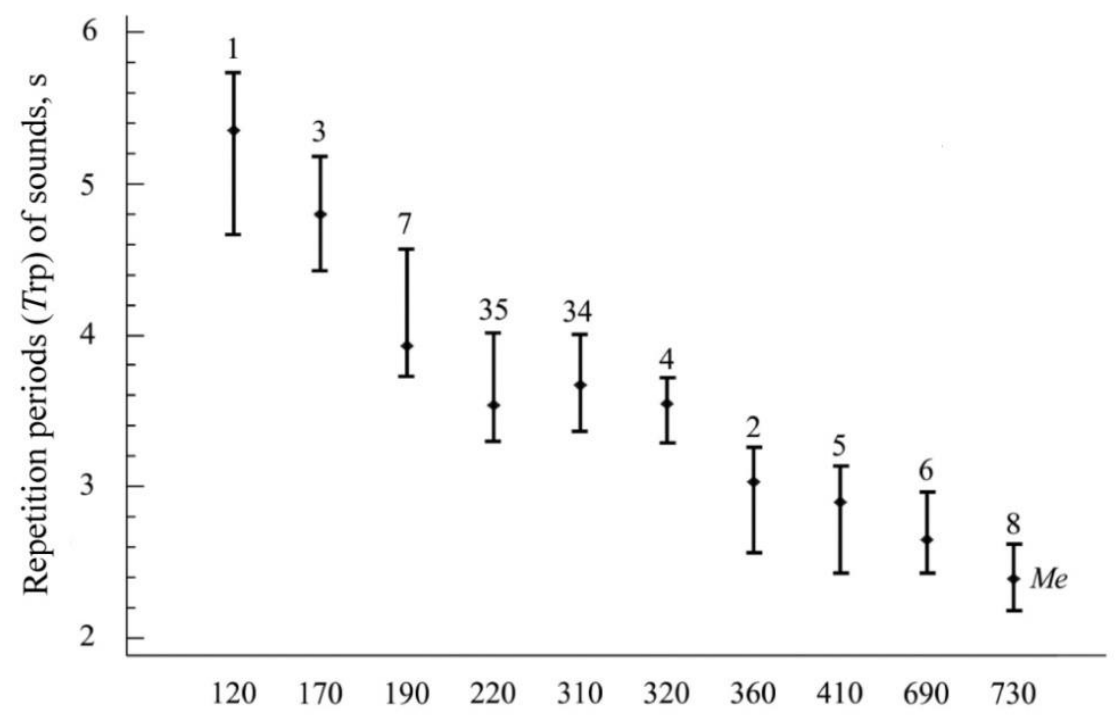

Habitat elevation of the population, $m$

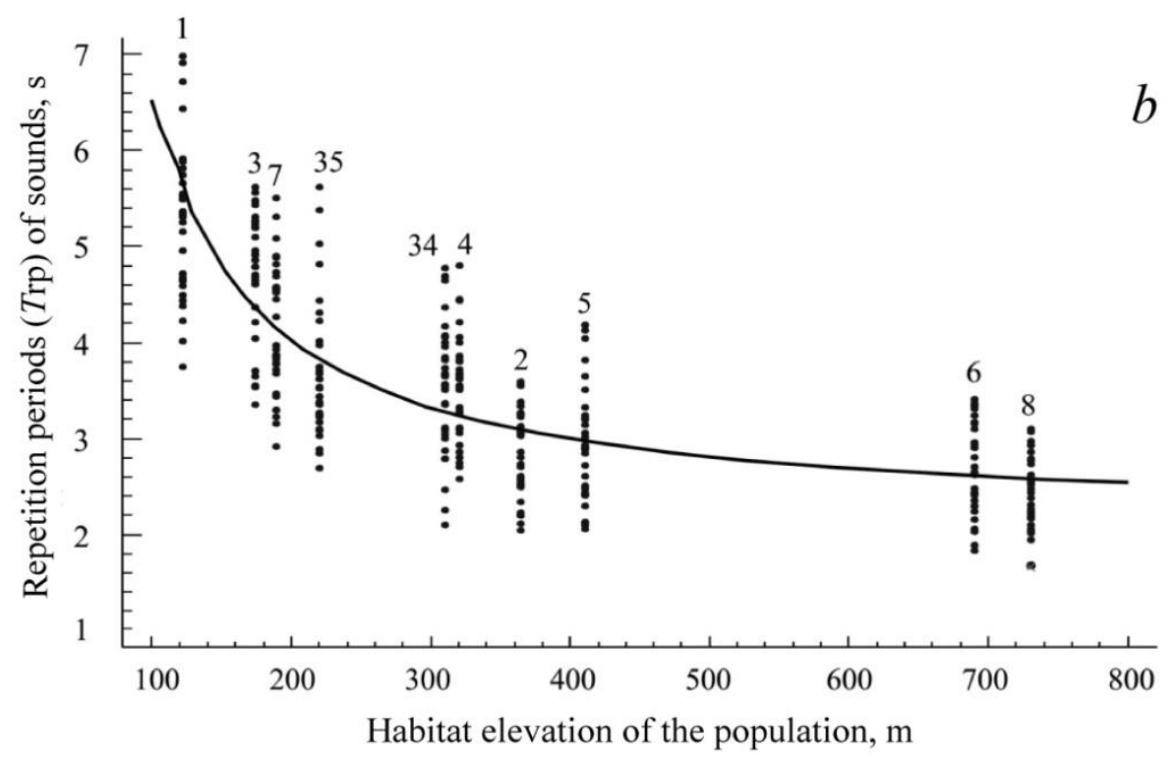

Fig. 4. Dependence of the repetition periods $\left(T_{\mathrm{rp}}\right)$ of sounds in the steppe marmot signal on the habitat elevation $(H)$ of the population (map see Fig. 2): $a$-median (Me) of repetition periods, with a $95 \%$ confidence interval; $b$ - repetition periods of sounds in local populations on the regression line. $1 \ldots$ 35 - registration numbers of populations.

The absence of statistically significant differences between the medians of neighboring populations (Fig.4:a) and the relatively loose distribution of the repetition periods (Fig.4 : $b$ ) can be explained by unaccounted factors, such as: gene flow softening natural selection, situational, individual, age, sex and seasonal changes in the signal, as well as the regional specificity of the relief.

The regression equation (1) makes it possible to construct a visual model of the variability of the repetition period of sounds depending on the habitat elevation of the population. Fig. 5 shows the sequences of sounds with the repetition periods calculated in 
the range of elevation from 100 to $700 \mathrm{~m}$. The timeline is limited to $20 \mathrm{~s}$. This time interval makes it possible to display the whole variety of variability of the rhythmic structure of the signal in the range of elevation distribution of the steppe marmot. If marmots are not provoked to flee, they can vocalize for tens of seconds. We know of a case, when the steppe marmot shouted at a person for about 5 minutes.

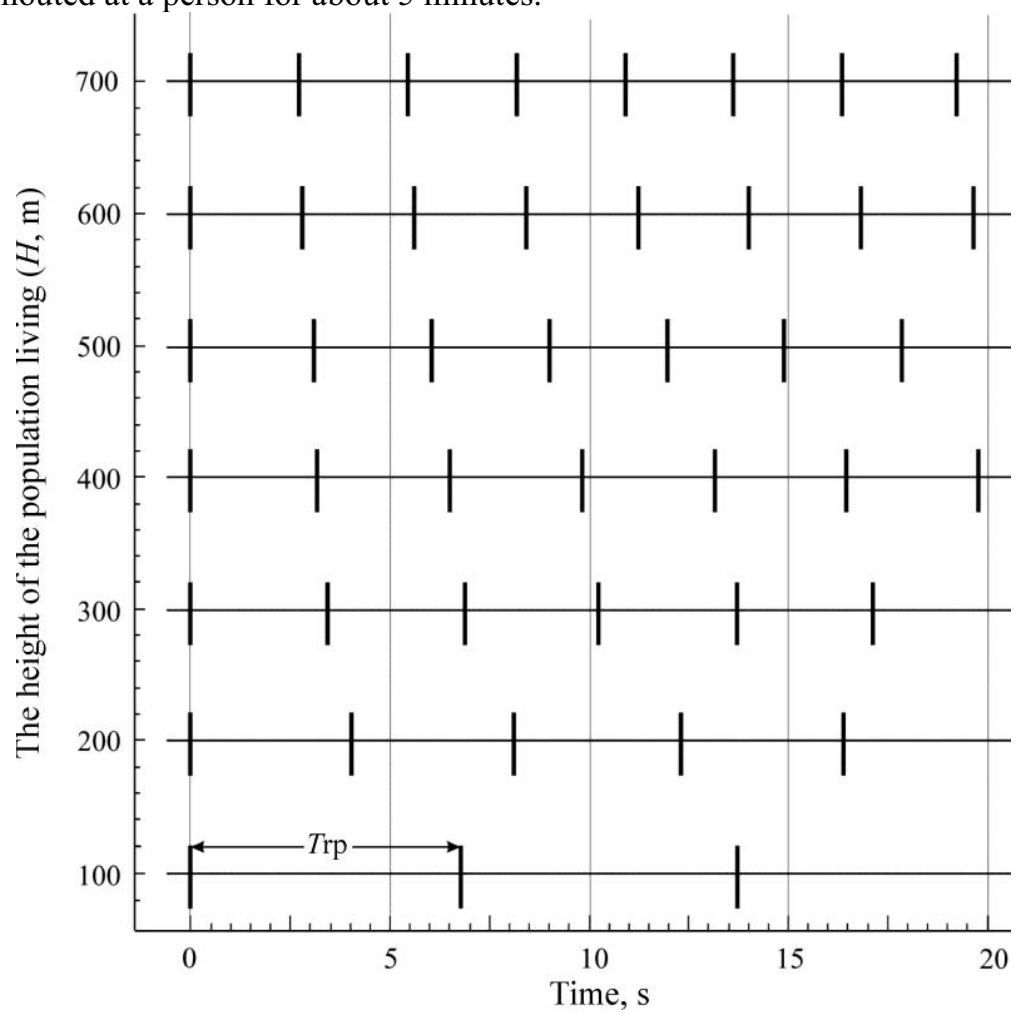

Fig. 5. Model of variability of the rhythmic structure of the steppe marmot' alarm call. The repetition period $\left(T_{r p}\right)$ of sounds in the signal is calculated relative to the habitat elevation $(H)$ of the population. Regression equations (1) see the text.

\section{Conclusion}

Our results confirm at the intraspecific level that the reactivity of marmots, realized through vocalization, is ecologically determined and that the natural selection factor is the terrain relief. Vocalization, as a manifestation of reactivity in response to visual perception of a stimulus, depends on the detection range of danger, limited by the properties of the relief in the habitats of local populations. An increase in the reactivity of marmots caused by an increase in the dismemberment of the relief suggests that the direct natural selection factor is the distance at which the signal source is most likely to detect a potentially dangerous stimulus.

Marmots, like many other inhabitants of open landscapes with daytime activity, have a specialized complex of adaptations that facilitate visual detection of danger at a great distance during daylight hours [9]. The high natural selective sensitivity of steppe marmots to the landscape structure is unexpected; although understandable - it is directed against factors that reduce the efficiency of this adaptive complex. Reducing the repetition periods of sounds (faster rhythm) compensates the signal source for the limited distance from which you can send a signal, feeling safe. 
The initial reaction that gave rise to a specialized form of vocalization is probably the escape of marmots into the burrow, accompanied by an alarm call [3, 4]. When the distance between a potentially dangerous stimulus (in our case, a human) and marmots becomes critical, they quickly run into the burrow with a series of rapidly following sounds (Fig. 1 $: e)$. This call is known in all species of marmots. Its rhythmic structure practically does not differ from the temporal organization of the alarm call of the inhabitants of the mountains (Fig. 1:c, $d$ ), the dismembered relief of which limits the view, which confirms the evolutionary-motivational continuity of these two vocal reactions.

Most likely, a complex of reactions to visual detection of danger controls the amygdala. This is indicated by its main functions [10,11]. According to David Amaral [10], the amygdala is a key component of a system that evaluates potential environmental hazards. If this is so, then we have the opportunity to investigate natural selection at the neural level, examining the variability of the amygdala in parallel with the variability of the reactivity of marmots.

Reactivity is one of the properties of temperament [12]. Therefore, our results can be interpreted as a natural selection of temperaments, as a result of which the plains, in comparison with the hills, inhabited by less temperamental populations. Following Wolf and Réale [2,12], I draw attention to our lack of knowledge about the evolution of ecologically determined animal behavior. The vocal activity of marmots can become a model object available for studying not only variability, but also factors of selection of ecologically determined behavior of animals in their natural habitats.

For help at different stages of the work, I thank K.I. Belovezhets, E.A. Vanisova, M.E. Goltsman, S.B. Pole (Republic of Kazakhstan), N.A. Formozov and M.V. Rutovskaya.

\section{References}

1. A.A. Nikol'skii, Auditory Signals of Mammals in the Evolutionary Process (Moscow, Nauka, 1984) [rus.]

2. M. Wolf, G. Sander van Doorn, F.J. Weissing, PNAS, 105, 41(2008)

3. A.A. Nikol'skii, Rus. J. Zoology, 53, 3(1974) [rus.]

4. A. Nikolski,Verhaltensbiologie. Internationales Symposium. 1983 / Zusammengestellt und bearbeitet von G.Tembrock, R.Siegmund und M.Nichelmann (HumboldtUniversitatzu Berlin, 1986)

5. I.M. Gromov, D.I. Bibikov, I.I. Kalabukhov, M.N. Meier, Fauna of the USSR. Mammals. V. III, 2. Terrestrial Sciurids (Marmotinae)(Moscow, Leningrad, Nauka, 1965) [rus.]

6. A.I. Spiridonov, Fundamentalofthegeneralmethodology of field geomorphological research andgeomorphological mapping(Moscow, Visshayashkola, 1970) [rus.]

7. S.S.Voskresenskiy, Dynamic geomorphology. Slope formation (Moscow, Moscow University, 1971) [rus.]

8. I.I. Schmalhausen, Factors of Evolution: The Theory of Stabilizing Selection (Blakiston, Philadelphia, 1949)

9. V.M. Smirin, O.Yu. Orlov, Priroda, 5 (1971) [rus.]

10. D.G. Amaral, Ann N Y Acad Sci., Dec., 1000 (2003).

11. A-S. Wiese, E.K.Needham, C.L.Noer, et al., Brain Structure and Function, 223, 4(2018)

12. D. Réale, S.M. Reader, D. Sol, et al., Biol. Rev., 82, 2 (2007). 